\title{
Radiomic assessment of the progression of acoustic neuroma after gamma knife stereotactic radiosurgery
}

\author{
Ganesh Narayanasamy*1, Geoffrey Zhang ${ }^{2}$, Eric Siegel ${ }^{3}$, Graham Campbell ${ }^{4}$, Eduardo G. Moros ${ }^{2}$, Edvaldo P. \\ Galhardo ${ }^{1}$, Steven Morrill ${ }^{1}$, John Day ${ }^{5}$, Jose Penagaricano ${ }^{1}$ \\ ${ }^{1}$ Department of Radiation Oncology, University of Arkansas for Medical Sciences, Arkansas, United States \\ ${ }^{2}$ H Lee Moffitt Cancer Center \& Research Institute, Tampa, Florida, United States \\ ${ }^{3}$ Department of Biostatistics, University of Arkansas for Medical Sciences, Little Rock, Arkansas, United States \\ ${ }^{4}$ College of Medicine, University of Arkansas for Medical Sciences, Little Rock, Arkansas, United States \\ ${ }^{5}$ Department of Neurosurgery, University of Arkansas for Medical Sciences, Little Rock, Arkansas, United States
}

Received: December 19, 2018

Accepted: March 14, 2019

Online Published: April 9, 2019

DOI: $10.5430 /$ jst.v9n2p1

URL: https://doi.org/10.5430/jst.v9n2p1

\begin{abstract}
Introduction: The aim of this study was to determine whether radiomic features measured at baseline in Magnetic Resonance images (MRI) of acoustic neuromas (AN) can predict Gamma Knife (GK) treatment outcome.

Methods: The study was conducted on pre- and post-GK MRI-T2 scans of 32 patients with AN who underwent stereotactic radiosurgery (SRS) for 12 Gy dose. Radiomic features extracted include Intensity, Fractals, Laplacian of Gaussian and textural Co-Occurrence, Run-length (RL), Size Zone, and Neighborhood Gray-Tone Difference matrices (NGTDM) features. Subjects were classified as treatment failures (TF) if tumor volume increased $>10 \%$. Pre- and post-SRS audiology reports were utilized in hearing evaluation.

Results: Fifteen subjects (47\%) qualified as TFs. In univariate receiver operating characteristic (ROC) analysis, two radiomic features, complexity in NGTDM and run percentage in RL, displayed areas under curves of $>0.65$.

Conclusion: This initial radiomic study establishes features that illustrates the prognostic ability of the SRS treatment in acoustic neuroma. Hearing preservation was achieved in a majority of acoustic neuroma patients treated in Gamma Knife.
\end{abstract}

Key Words: Radiomics, SRS Radiomics, Acoustic neuroma, vestibular schwannoma, Gamma Knife

\section{INTRODUCTION}

Acoustic neuroma (AN) or vestibular schwannoma was reported in 5\%-8\% of all intracranial tumors are slowly progressing benign growth that develops on the eighth cranial nerve, causing gradual hearing loss, loss of balance, and facial numbness. ${ }^{[1]}$ Contrast-enhanced magnetic resonance (MR) images or computed tomography (CT) are considered the gold-standard imaging for periodic assessment, diagnosis and staging of AN. Treatment options such as microsurgery and craniotomy were associated with morbidities. ${ }^{[2]}$ For patients with contraindications to surgery, stereotactic radiosurgery (SRS) is an alternative option. Noren et al. were the first to report favorable outcomes with Gamma Knife (GK)based SRS treatment. ${ }^{[3]}$ High dose conformality and high dose heterogeneity with SRS might suggest higher tumor control rate with less tumor morbidities ${ }^{[4]}$ and low incidence

*Correspondence: Ganesh Narayanasamy; Email: ganesh@uams.edu; Address: Department of Radiation Oncology, University of Arkansas for Medical Sciences, Slot 771, 4301 W Markham St, Little Rock, AR 72205, United States. 
of neuropathy with SRS. ${ }^{[5]}$ Prevention of hearing deterioration following SRS is critical for long-term hearing preservation. Although underpowered, maximum radiation dose to cochlear nucleus was shown to be single significant prognostic factor for hearing deterioration. ${ }^{[6]} \mathrm{WHO}$ and RECIST criteria for response is often based on 1D tumor size. ${ }^{[7,8]} \mathrm{Al}-$ though response to therapy is measurable, these descriptors fall short of determining treatment outcome. ${ }^{[9]}$ Although sequential MRI scans are preferred, increase in tumor volume after SRS is not considered as treatment failure and transient changes were observed by Pollock et al. ${ }^{[10]}$ Based on imaging methodology, $2 \mathrm{~mm}$ diameter or $10 \%$ volume change is considered significant. ${ }^{[11]}$ Radiomics aid extracting quantitative features from medical images. ${ }^{[12]}$ The present study is an effort to use radiomics analysis to quantify MR images of AN patients treated with SRS at our institution. The purpose of this study is to examine prognostic property of radiomic features in pre-SRS MR images to treatment outcome. Audiometric classifications such as Gardner-Robertson hearing scale (GRHS) was used to grade hearing before and after treatment of AN. ${ }^{[13]}$

\section{METHODS AND MATERIALS}

\subsection{Patient population}

In this Institutional Review Board approved study, data from 32 patients diagnosed with $\mathrm{AN}$ and treated using singlefraction SRS on a GK Perfexion (Elekta Instruments AB, Stockholm, Sweden) at our institution between 2010 and mid-2018 were included. A tumor volume increase larger than $10 \%$ following GK is considered treatment failure (TF)

\subsection{Treatment planning}

The treatment planning was performed on MRI-T2 images acquired at sub-mm slice thickness in a $1.5 \mathrm{~T}$ Philips Achieva MRI scanner (Philips Healthcare, Best, The Netherlands) using Leksell GammaPlan Ver 10.0 in TMR10 algorithm. Gross tumor volume (GTV) is contoured on each slice of MR images. A single fraction stereotactic radiation dose of 12 Gy to the $50 \%$ isodose volume was prescribed to the GTV. Tolerance dose to normal structures were observed, and hot spots were restricted within the target volume.

\subsection{Radiomic extraction}

Mathematical definitions of all radiomic features, and the extraction methods, were previously described. ${ }^{[14]}$ MR images were exported to a MimVista software version 6.6.5 (MIM Software Inc., Cleveland, OH). MR and target contour were exported to an in-house software for feature extraction. Fiftyfive three-dimensional (3D) radiomic features extracted from 3D MR images quantify tumor characteristics, including features based on intensity, texture, shape, fractal dimen- sions, and Laplacian of Gaussian (LoG). While 3D image intensity was derived from first order statistics, 3D textural features were extracted from gray-level co-occurrence matrix (GLCM), gray-level run-length matrix (GLRLM), gray-level size zone matrix (GLSZM), and neighborhood gray-tone difference matrices (NGTDM). These 4 matrices used in feature extraction were based on averages along 13 directions on $3 \mathrm{D}$ image space, as stated in Kurani et al. ${ }^{[15]}$ Oliver et al. had described extraction of GLCM features that deal with spatial dependence of neighboring voxels. ${ }^{[16]}$ GLRLM feature implementation followed guidelines published elsewhere. ${ }^{[17]}$ GLSZM and NGTDM features were developed based on Thibault et al. ${ }^{[18]}$ and Amadasun et al., ${ }^{[19]}$ respectively. Shape features describe 3D geometric properties of the tumor. ${ }^{[20]}$ Fractal dimensions were calculated based on Sarkar et al. ${ }^{[21]}$ LoG features were determined using filters of sizes ranging from $0.5 \mathrm{~mm}$ to $2 \mathrm{~mm}$ in increments of 1 $\mathrm{mm} .{ }^{[22]}$ Image intensities were binned into 64 equally spaced gray levels for radiomic feature extraction in an in-house image analysis software.

\subsection{Statistical analysis}

Univariate Receiver Operating Characteristics (ROC) analysis was performed on each Radiomic feature measured at baseline (shortly before SRS treatment) using the Logistic Procedure in SAS v9.4 software (The SAS Institute, Cary, NC, USA), in order to summarize the prognostic performance over a range of trade-offs between rates of truepositive and false-positive TF predictions. Because there were fewer than 20 TFs, multivariate analysis was not conducted in order to avoid over-fitting. ${ }^{[23]}$ An unadjusted alpha $=0.05$ significance level was used for all hypothesis tests despite the multiple testing, in order not to inflate Type II error in this small, modestly powered study. Performance of a radiomic feature at successfully identifying a TF was measured using area under curve (AUC) in the ROC curve.

\subsection{Hearing evaluation}

The hearing evaluation before and after GK treatment was based on audiogram. A hearing sensitivity measure, puretone average (PTA) was extracted from hearing levels (decibels) at three frequency levels - 500, 1000 and $2000 \mathrm{~Hz}$. Word recognition score (WRS) was used as a measure of the speech audiometry. In GRHS classification, hearing ability was assessed from PTA and WRS on a scale of 1 - 5 (1-good, 2-serviceable, 3-non-serviceable, 4-poor, and 5deaf). ${ }^{[24]}$ Hearing preservation was defined as maintenance of GRHS grades 1-2 with SRS treatment. The audiogram reports were available both prior to SRS and post-SRS on 14 of 32 cases due to patient compliance. 


\section{Results}

\subsection{Patient population}

Among the 32 AN patients included in this study, seventeen patients were female and 15 patients were male. The mean \pm standard deviation (SD) age at SRS were $59 \pm 13$ years. The mean \pm SD time interval between MRI examinations before and after SRS was $207 \pm 72$ days (range: 88 - 368 days). AN was localized on the right vestibular nerve on 14 patients, on the left vestibular nerve on 18 patients and none bilaterally. Figure 1 displays MR images of a typical AN on the left ear acquired pre-GK and on 16th month follow-up visit post-GK.

\subsection{Tumor volume}

Tumor was contoured in MRI scans acquired before and after SRS. Based on pre-SRS tumor volume, 22 can be considered small ( $<1.5 \mathrm{~cm}$ equivalent diameter) and 10 were considered medium-sized (equivalent diameter between 1.5 and 2.5 $\mathrm{cm})$. The average \pm standard deviation (SD) of the tumor size was $1.5 \pm 1.35 \mathrm{cc}$ prior to SRS and $1.7 \pm 1.2 \mathrm{cc}$ post SRS. The absolute tumor volume change was minimal in almost all cases. Shown in Figure 2 is the percent change in PTV against pre-GK PTV classified according to the type of treatment response.

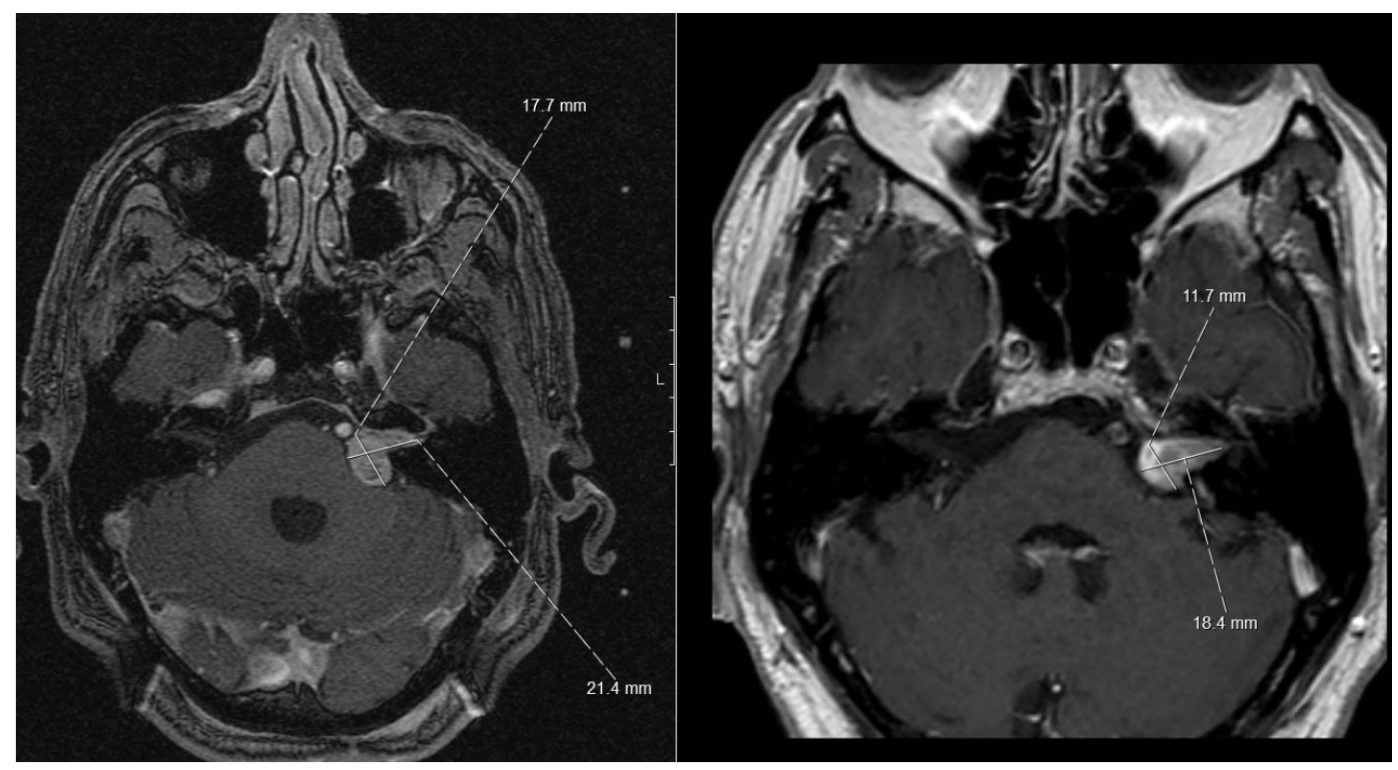

Figure 1. MR images revealing a typical AN in the left ear (A) in pre-GK and (B) in 16 month follow up visit post-GK

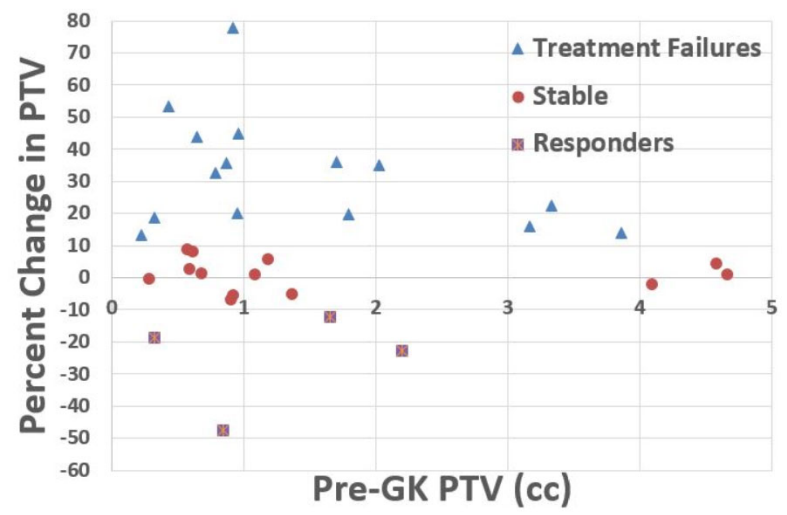

Figure 2. Percent change in PTV against the pre-GK PTV classified by the treatment response

With regard to the change in tumor size on MR images following SRS, Vokurka et al estimated inaccuracies of $\pm 13 \%$ in manual segmentation. ${ }^{[25]}$ In our study, a volume change $>$ $\pm 10 \%$ have been considered as progression or regression, respectively. A sizeable number $(\mathrm{N}=15$ or $47 \%)$ can be classified as treatment failures. Among the other $53 \%$ cases $(\mathrm{N}$ $=17$ ), 13 cases had $<10 \%$ volume change in either direction (stable lesions) while only 4 cases showed decline $>10 \%$ in tumor volume (responders). In Vokurka et al, tumor growth was shown in $12(32 \%)$, potential growth for $7(18 \%)$, no growth for $17(45 \%)$ and shrinkage only for 2 cases $(5 \%) .{ }^{[25]}$

\subsection{ROC analysis}

In ROC curve analysis, 2 features with highest AUC values are complexity in NGTDM and run percentage (RPC) in GLRLM. Amadusan et al had described complexity as a visual information content of a texture derived from NGTDM19. RPC was computed from ratio of the total number of runs of all gray scale values to total number of pixels in the image. ${ }^{[17]}$ Figure 3 illustrates the ROC curve of the complexity and RPC. Their ROC AUCs $(90 \%$ confidence limits) are $0.729(0.571-0.888)$ for Complexity and 0.682 
(0.522 - 0.842) for RPC. The fact that the lower $90 \%$ confidence limits of AUCs of these 2 Radiomic features identified were $>0.5$ shows that the ROC AUCs were significantly greater than the null AUC value of 0.5 via 1 -sided test at $\alpha=$ 0.05 .
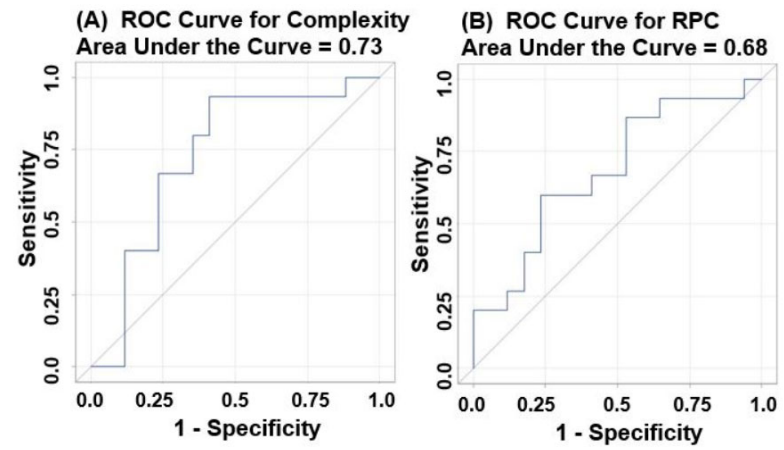

Figure 3. Receiver operating characteristics (ROC) curve for (A) complexity and (B) RPC. The ROC AUCs $(90 \%$ confidence limits) are $0.73(0.57-0.89)$ for Complexity and $0.68(0.52-0.84)$ for RPC

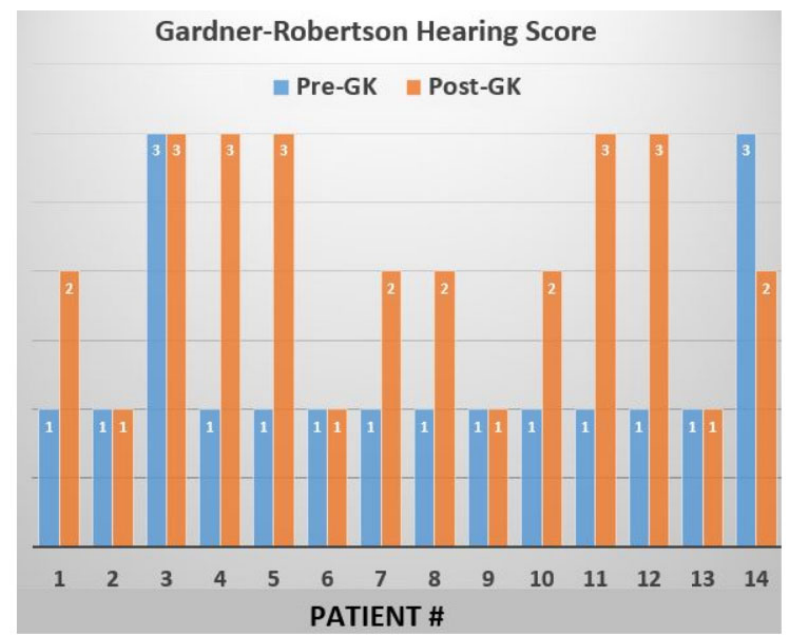

Figure 4. Gardner-Robertson hearing scores from a subset of patients with audiogram reports acquired before and after GK-based SRS treatment

\subsection{Hearing evaluation}

The average \pm SD of GRHS values were $1.3 \pm 0.7$ prior to SRS, and were $2.1 \pm 0.8$ post SRS treatment, as shown in Figure 4. The $\mathrm{X}$-axis in Figure 4 indicate the patient \# with audiogram reports obtained before and after SRS treatment. Before GK, 12 patients (86\%) evaluated were in scale 1 and $2(14 \%)$ in scale 3 . Hearing was preserved in 8 of 14 patients $(57 \%)$ who showed GRHS grades of 1-2 both prior to and post-SRS treatment. Iwai et al. reported that useful hearing was preserved in only $56 \%$ of patients after GK SRS, although good tumor control rate was observed in 96\%. ${ }^{26]}$
Ten out of 14 patients had less than $20 \mathrm{~dB}$ loss in PTA in the follow-up examination.

\section{Discussion}

A PubMed search with keywords such as "Radiomics" and "Acoustic Neuromas" or "Vestibular schwannoma" failed to return any tangible result. This study could be the first one on radiomics based prognosis of a benign tumor. In this study, we have investigated a total of 55 radiomic features using univariate ROC analysis for treatment failure prognosis following SRS of ANs. Reasonably good performance at predicting treatment failure was achieved for 2 features out of 55, the AUCs of which exceeded a threshold of 0.65 with a lower $90 \%$ confidence intervals higher than 0.5 . Absence of sequential MRI scans and dependence on a single follow-up MRI scan can be a drawback of the study. A long term study with sequential MRI scans may have helped in identification of pseudo-progression in tumor volumes after SRS. It is debatable if some of the tumor volume change is transient, as discussed at length by Pollock et al. ${ }^{[27]}$ However, this aspect which involves serial imaging is beyond the scope of our study. An inherent bias due to limited size of patient population is present in our study, like in any study with similar patient size. A study on evaluation of radiation associated ototoxicity was presented by Bhandare et al. ${ }^{[28]} \mathrm{A}$ specific tolerance radiation dose to cochlea resulting in ototoxicity was not determined, mainly due to the small volume of cochlea where a dose volume histogram is not feasible. Hua et al observed the positive correlation between increases in cochlea dose with hearing loss from conformal radiotherapy of pediatric brain tumors. ${ }^{[29]}$ Kano et al. revealed that a cochlea dose less than 4.2 Gy resulted in retention of serviceable hearing at 2 years post GK-based SRS of ANs. ${ }^{[30]}$ Hearing evaluation was pursued based on the hearing status before and after SRS treatment using the Gardner-Robertson hearing score. Based on the available audiogram test performed before and after treatment, hearing preservation was achieved in a majority of our patients.

\section{Conclusion}

Our study is an initial attempt to investigate the prognostic potential of radiomics of acoustic neuromas treated with radiosurgery. Two radiomic features were identified using logistic regression modelling for prognosis of radiosurgery treatment.

\section{ACKNOWLEDGEMENTS}

The authors would like to thank Ms Karen Baxter-Rhoades, University of Arkansas for Medical Sciences for assistance in audiogram data collection. 


\section{REFERENCES}

[1] Stangerup SE, Caye-Thomasen P. Epidemiology and natural history of vestibular schwannomas. Otolaryngol Clin North Am. 2012; 45(2): 257-268. PMid:22483814. https ://doi.org/10.1016/j . otc. 2011.12 .008

[2] Wiegand DA, Fickel V. Acoustic neuroma-the patient's perspective: Subjective assessment of symptoms, diagnosis, therapy, and outcome in 541 patients. Laryngoscope. 1989; 99: 179-187. PMid:2913427.

[3] Noren G, Greitz D, Hirsch A, et al. Gamma knife surgery in acoustic tumours. Acta Neurochir Suppl (Wien). 1993; 58: 104-107. https ://doi .org/10.1007/978-3-7091-9297-9_24

[4] Andrews DW, Suarez O, Goldman HW, et al. Stereotactic radiosurgery and fractionated stereotactic radiotherapy for the treatment of acoustic schwannomas: comparative observations of 125 patients treated at one institution. Int J Radiat Oncol Biol Phys. 2001; 50(5): 1265-1278. https : //doi.org/10.1016/S0360-3016(01 ) 01559-0

[5] Flickinger JC, Kondziolka D, Pollock BE, et al. Evoluton in technique for vestibular schwannoma radiosurgery and effect on outcome. International Journal of Radiation Oncoilgoy Biology Physics. 1996; 36(2): $275-280$

[6] Paek SH, Chung HT, Jeong SS, et al. Hearing preservation after gamma knife stereotactic radiosurgery of vestibular schwannoma. Cancer. 2005; 104(3): 580-590. PMid:15952200. https : //doi.org/10.1002/cncr.21190

[7] WHO (1979) Handbook for Reporting Results of Cancer Treatment Offset Publication No. 48. Geneva: World Health Organization.

[8] Therasse P, Arbuck SG, Eisenhauer EA, et al. New guidelines to evaluate the response to treatment in solid tumors. European Organization for Research and Treatment of Cancer, National Cancer Institute of the United States, National Cancer Institute of Canada. J Natl Cancer Inst. 2000; 92: 205-216. PMid:10655437. https://doi.org/10.1093/jnci/92.3.205

[9] Blakeley JO, Evans DG, Adler J, et al. Consensus recommendations for current treatments and accelerating clinical trials for patients with neurofibromatosis type 2. American Journal of Medical Genetics Part A. 2012; 158(1): 24-41.PMid:22140088. https : //doi.org/10.1002/ajmg.a.34359

[10] Pollock BE, Driscoll CL, Foote RL, et al. Patient outcomes after vestibular schwannoma management: a prospective comparison of microsurgical resection and stereotactic radiosurgery. Neurosurgery. 2006; 59(1): 77-85. PMid:16823303. https://doi.org/10.122 7/01. NEU.0000219217.14930.14

[11] Timmer FC, Hanssens PE, van Haren AE, et al. Follow-up after gamma knife radiosurgery for vestibular schwannomas. The Laryngoscope. 2011; 121(7): 1359-1366. https ://doi.org/10.1002/ $\operatorname{lary} .21763$

[12] Aerts HJ, Velazquez ER, Leijenaar RT, et al. Decoding tumour phenotype by noninvasive imaging using a quantitative radiomics approach. Nature communications. 2014. https : //doi .org/10.1038/ncom ms5006

[13] Saadatmand HJ, Cheng-Chia W, Wang TJC. Interdisciplinary management of acoustic neuromas. Appl Rad Oncol. 2016; 5(1): 5-16.

[14] Shafiq M, Zhang GG, Latifi K, et al. Intrinsic dependencies of CT radiomic features on voxel size and number of gray levels. Medical physics. 2017; 44(3): 1050-1062. PMid:28112418. https : //doi.org/10.1002/mp.12123
[15] Kurani AS, Xu DH, Furst J, et al. Co-occurrence matrices for volumetric data. Heart. 2004; 27: 25.

[16] Oliver JA, Budzevich M, Zhang GG, et al. Variability of Image Features Computed from Conventional and Respiratory-Gated PET/CT Images of Lung Cancer. Translational Oncology. 2015; 8: 524-534 PMid:26692535. https://doi.org/10.1016/j.tranon. 2015 11.013

[17] Galloway MM. Texture analysis using gray level run lengths. Computer Graphics and Image Processing. 1975; 4: 172-179. https: //doi .org/10.1016/S0146-664X (75)80008-6

[18] Thibault G, Fertil B, Navarro C, et al. Texture indexes and gray level size zone matrix application to cell nuclei classification. 2009.

[19] Amadasun M, King R. Textural features corresponding to textural properties. IEEE Transactions on System on Man and Cybernetics. 1989; 19: 1264-1274. https://doi.org/10.1109/21.44046

[20] Kumar V, Gu Y, Basu S, et al. Radiomics: the process and the challenges. Magnetic Resonance Imaging. 2012; 30: 1234-1248. PMid:22898692. https://doi.org/10.1016/j.mri.2012.06. 010

[21] Sarkar N, Chaudhuri BB. An efficient approach to estimate fractal dimension of textural images. Pattern Recognition. 1992; 25: 1035 1041. https://doi.org/10.1016/0031-3203(92)90066-R

[22] Ganeshan B, Panayiotou E, Burnand K, et al. Tumour heterogeneity in non-small cell lung carcinoma assessed by $\mathrm{CT}$ texture analysis: A potential marker of survival. Eur Radiol. 2012; 22: 796802. PMid:22086561. https ://doi.org/10.1007/s00330-011 $-2319-8$

[23] Peduzzi P, Concato J, Kemper E, et al. A simulation study of the number of events per variable in logistic regression analysis. Journal of Clinical Epidemiology. 1996; 49(12): 1373-1379. https : //doi.org/10.1016/S0895-4356(96)00236-3

[24] Sweeney AD, Carlson ML, Ehtesham M, et al. Surgical approaches for vestibular schwannoma. Current Otorhinolaryngology Reports. 2014; 2(4): 256-264. https://doi.org/10.1007/s40136-014 $-0060-\mathrm{y}$

[25] Vokurka EA, Herwadkar A, Thacker NA, et al. Using Bayesian tissue classification to improve the accuracy of vestibular schwannoma volume and growth measurement. American Journal of Neuroradiology. 2002; 23(3): 459-467. PMid:11901019.

[26] Iwai Y, Yamanaka K, Shiotani M, et al. Radiosurgery for acoustic neuromas: results of low-dose treatment. Neurosurgery. 2003; 53: 282-288. PMid:12925242. https://doi.org/10.1227/01.NEU $.0000073416 .22608 . \mathrm{B} 3$

[27] Pollock BE. Management of vestibular schwannomas that enlarge after stereotactic radiosurgery: treatment recommendations based on a 15 year experience. Neurosurgery. 2006; 58: 241-248. PMid:16462477. https://doi.org/10.1227/01.NEU.0000194 833.66593.8B

[28] Bhandare N, Jackson A, Eisbruch A, et al. Radiation therapy and hearing loss. International Journal of Radiation Oncology. Biology, Physics. 2010; 76(3 Suppl): S50-S57. https ://doi .org/10.101 6/j.ijrobp.2009.04.096

[29] Hua C, Bass JK, Khan R, et al. Hearing loss after radiotherapy for pediatric brain tumors: effect of cochlear dose. International Journal of Radiation Oncology Biology Physics. 2008; 72(3): 892-899.

[30] Kano H, Kondziolka D, Khan A, et al. Predictors of hearing preservation after stereotactic radiosurgery for acoustic neuroma. Journal of Neurosurgery. 2009; 111(4): 863-873 